\title{
Paper
}

Int'I J. of Aeronautical \& Space Sci. 14(2), 193-199 (2013)

DOI:10.5139/IJASS.2013.14.2.193

\section{Earth Albedo perturbations on Low Earth Orbit Cubesats}

\author{
N. S. Khalifa* \\ Mathematics Department, Deanship of Preparatory Year- Girls Branch, University of Hail (UOH), KSA. \\ Space Research Lab, National Research Institute of Astronomy and Geophysics (NRIAG), Egypt.
}

\section{T.E. Sharaf-Eldin**}

Mathematics Department, Deanship of Preparatory Year- Girls Branch, University of Hail (UOH), KSA. Electrical. Eng. Dept, Faculty of Engineering, Alexandria University, Egypt.

\begin{abstract}
This work investigates the orbital perturbations of the cubesats that lie on LEO due to Earth albedo. The motivation for this paper originated in the investigation of the orbital perturbations for closed- Earth pico-satellites due to the sunlight reflected by the Earth (the albedo). Having assumed that the Sun lies on the equator, the albedo irradiance is calculated using a numerical model in which irradiance depends on the geographical latitude, longitude and altitude of the satellite. However, in the present work the longitude dependency is disregarded. Albedo force and acceleration components are formulated using a detailed model in a geocentric equatorial system in which the Earth is an oblate spheroid. Lagrange planetary equations in its Gaussian form are used to analyze the orbital changes when $\mathrm{e} \neq 0$ and $i \neq 0$. Based on the Earth's reflectivity data measured by NASA Total Ozone Mapping Spectrometer (TOMS project), the orbital perturbations are calculated for some cubesats. The outcome of the numerical test shows that the albedo force has a significant contribution on the orbital perturbations of the pico-satellite which can affect the satellite life time.
\end{abstract}

Key words: cubesat specifications, terrestrial albedo, NASA Total Ozone Mapping Spectrometer (TOMS Project), radiative force, geocentric equatorial coordinate systems, Lagrange planetary equations.

\section{Introduction}

Recently, there is significant interest in developing a new class of standardized pico-satellites called cubesats. Cubesats have been the best choice for universities and research institutes toward starting space technology developments. The mass of the cubesat is restricted to be $1 \mathrm{~kg}$ and its size is confined to be $.1 \mathrm{~m}$ on all sides. This small size is considered as the main advantage of cubesats which enable them to be launched as an auxiliary payload and result in less cost and test requirements. The surface of the cubesat is covered by six aluminum plates, each of which has a solar chip attached to it. The majority of these satellites are placed in a low Earth sun synchronous orbit (the inclination is close to $98^{\circ}$ ) [1] and [11]
Many factors affect the satellite orbital motion. The Earth gravitational field has the master role in perturbing the satellite dynamical motion. However, the non-gravitational factors (e.g. solar radiation pressure, air drag, luni-solar attraction... etc) play a significant role in perturbing the orbital motion. Several authors have performed a suite of studies and models to illustrate those effects on various types of satellites and orbits. Over the last few years, a vast knowledge of natural radiation pressure effects on satellite dynamics and rotation has been achieved. The main contribution of the natural radiation pressure is due to the direct solar radiation so a variety of models were constructed to estimate their force [2], [3], [9], [11], and [19]. The second main contribution of radiation forces is due to the Earth reflected radiation known
This is an Open Access article distributed under the terms of the Creative Commons Attribution Non-Commercial License (http://creativecommons.org/licenses/by$\mathrm{nc} / 3.0 /$ which permits unrestricted non-commercial use, distribution, and reproduction in any medium, provided the original work is properly cited. cc * Associate Professor, Corresponding Author : Asmaa_2000_2000@yahoo.com ** Associate Professor 
as the albedo. It is an extremely complex phenomenon which shows relevant spatial and temporal variations. Albedo depends upon the reflectivity of the illuminated surface of the Earth that is visible to the spacecraft, the solar angle, and the position of the spacecraft in space. Moreover, it depends on seasonal variations and geographical longitude and latitude of the Earth surface that is illuminated by the Sun and seen by the satellite [4], [6] and [17].

Based on a new numerical modeling of the albedo in which the irradiance is calculated depending on the geographical latitude, longitude and altitude of the satellite, the main issue of this work is to investigate the orbital perturbations of the cubesats lying on LEO due to Earth albedo.

\subsection{The Albedo Irradiance}

The albedo irradiance, which reaches the satellite surface, is determined using a numerical model. This model is based on partitioning the Earth surface into a number of cells forming a grid. Then the incident solar irradiance on each cell is used to calculate the total radiant flux. The total albedo irradiance, $S$, at the satellite surface is given by [4] and [6]:

$$
S= \begin{cases}\frac{\sigma\left(\varphi_{g}, \theta_{g}\right) E_{A M 0} A_{C}\left(\hat{\rho}_{\text {sun }}^{T} \hat{n}_{C}\right)\left(\hat{\rho}_{\text {sat }}^{T} \hat{n}_{C}\right)}{\pi\left|\bar{\rho}_{\text {Sat }}\right|^{2}} & \text { if }\left(\varphi_{g}, \theta_{g}\right) V_{\text {Sim }} \cap V_{\text {Sat }} \\ 0 & \text { else }\end{cases}
$$

where $v_{\text {Sum }} \cap v_{\text {sat }}$ is the set of grid points that are illuminated by the Sun and visible by the satellite, $\sigma\left(\varphi_{g}, \theta_{g}\right)$ is the reflectivity of the grid points of latitude $\varphi_{g}$ and longitude $\theta_{g}, E_{A M 0}=1367 \mathrm{~W} / \mathrm{m}^{2}$ is the incident solar irradiance $\hat{n}_{C}$ is the unit vector normal to the grid cell and $\rho_{\text {sun }}$ are $\rho_{\text {set }}$ unit vectors directed from the grid center to the Sun and satellite , respectively. The cell area $A_{C}\left(\varphi_{g}\right)$ is found using the surface revolutions as in [4] and [6]:

$$
A_{C}\left(\varphi_{g}\right)=\theta_{g} r_{E}^{2}\left[\cos \left(\varphi_{g}-\frac{\Delta \varphi_{g}}{2}\right)-\cos \left(\varphi_{g}+\frac{\Delta \varphi_{g}}{2}\right)\right],
$$

where $\Delta \theta_{g}=1.25^{\circ}, \Delta \varphi_{g}=1^{\circ}$ and $r_{E}$ is the Earth mean radius.

It was obvious that the albedo irradiance had a large dependency on the geographical latitude of the grid points. The maximum albedo is observed over the poles and decreased by moving away from them and towards the shadow side of the Earth. Moreover, there is a significant dependency on the longitude and the solar angle, where albedo has maximum values for low solar angles [6], [16] and [17]. However, as previously mentioned, the longitude dependency is disregarded in this work.

These equations represent the albedo contribution of a single Earth cell. However, the sunlit area visible to the satellite is decomposed into a definite number of cells. So, the total albedo irradiance reaching to the satellite can be obtained by summing up the contribution of each cell [6] and [17]:

$$
S_{\text {total }}=\sum_{i=1}^{k} S_{i}
$$

where $k$ is defined as the number of illuminated Earth cells visible from the satellite.

\subsection{The Albedo Force.}

The total radiant force exerted on a flat non-perfectly reflecting surface is given by [13]:

$$
\bar{f}=f \hat{m}
$$

where,

$$
\begin{aligned}
f & =\frac{S A}{C}\left[4 \rho^{\prime} \beta \cos ^{4} \eta+2\left(1+\rho^{\prime} \beta\right)\left(B_{f} \rho^{\prime}(1-\beta)+\alpha^{\prime} \frac{\varepsilon_{f} B_{f}-\varepsilon_{b} B_{b}}{\varepsilon_{f}+\varepsilon_{b}}\right) \cos ^{3} \eta+\right. \\
& \left.+\left\{\left(B_{f} \rho^{\prime}(1-\beta)+\alpha^{\prime} \frac{\varepsilon_{f} B_{f}-\varepsilon_{b} B_{b}}{\varepsilon_{f}+\varepsilon_{b}}\right)^{2}+\left(1-\rho^{\prime} \beta\right)^{2}\right\} \cos ^{2} \eta\right]^{1 / 2}
\end{aligned}
$$

where $\hat{m}$ is a unit vector directed through the force direction, $c$ is the speed of light and $S$ is the radiation irradiance at the satellite surface, $\eta$ is the radiation incident angle to the satellite surface normal, $\beta$ is the satellite surface specularity, $\rho^{\prime}$ is the satellite surface reflectivity, $B_{f}$ and $B_{b}$ are the nonLambartian coefficient of the front and back surfaces of the spacecraft, respectively, $\alpha^{\prime}$ is the spacecraft absorption coefficient, and $\varepsilon_{f}$ and $\varepsilon_{b}$ are the front and back satellite surface emissivity, respectively.

For non-perfectly reflecting surfaces, the force vector, $\hat{m}$ , is not directed normal to the surface. But, it inclines by an angle $\vartheta$, known as the cone angle, to the incident direction as depicted in figure 1 . So, the force components in the incident direction will be:

$$
\bar{f}_{\rho}=f \cos \vartheta \hat{\rho}
$$

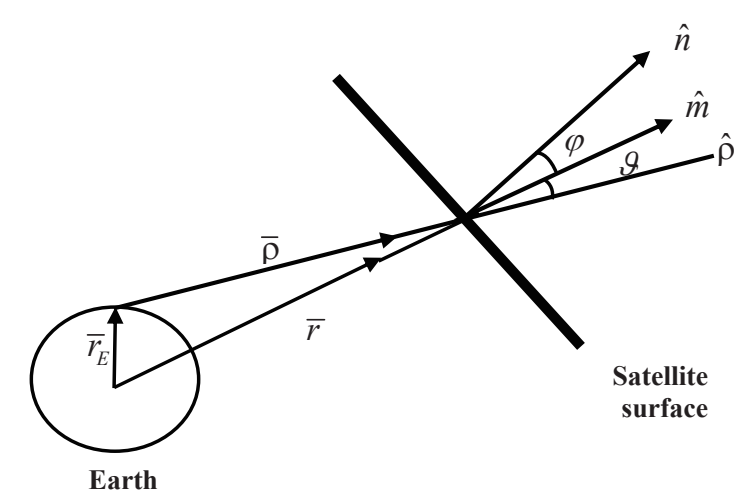

Fig. 1. Albedo intensity delivered to the satellite surface 


\subsection{The coordinate systems}

The geocentric equatorial system with $\hat{e}_{x}$ directed parallel to the Earth equatorial plane, $\hat{e}_{v}$ directed in the plane that contains the meridian of the sub-satellite point and $\hat{e}_{z}$ directed normal to the equatorial plane is used. As shown in Fig. 1, the vector directed through incident radiation, $\hat{\rho}_{\text {sat }}$ , is given by:

$$
\bar{\rho}_{\text {sat }}=\bar{r}-\bar{r}_{E},
$$

where $\bar{r}$ is the satellite position vector and $\bar{r}_{E}$ is the Earth radius vector. For oblate Earth, the Earth radius vector is given by [7]:

$$
\bar{r}_{E}=\left(\begin{array}{l}
G_{1} \cos \varphi_{g} \cos \theta \\
G_{1} \cos \varphi_{g} \sin \theta \\
G_{2} \sin \varphi_{g}
\end{array}\right),
$$

with

$$
G_{1}=\frac{a_{e}}{\left(1-\left(2 f_{e}^{\prime}-f_{e}^{\prime 2}\right) \sin ^{2} \varphi_{g}\right)^{1 / 2}}+h, \quad G_{2}=\frac{a_{e}\left(1-f_{e}^{\prime}\right)^{2}}{\left(1-\left(2 f_{e}^{\prime}-f_{e}^{\prime 2}\right) \sin ^{2} \varphi_{g}\right)^{1 / 2}}+h(9)
$$

where $a_{e}$ is the Earth's Equatorial radius, $f_{e}^{\prime}$ is the Earth's flattening, $\varphi_{g}$ is the geodetic latitude of the grid point, $h$ is the height above sea level and $\theta$ is the sidereal time.

The satellite position vector, $\bar{r}$, in the geocentric coordinate system, is given by [7]:

$$
\bar{r}=r\left(\begin{array}{l}
\cos \Omega \cos (\omega+v)-\sin \Omega \sin (\omega+\nu) \cos i \\
\sin \Omega \cos (\omega+\nu)+\cos \Omega \sin (\omega+\nu) \cos i \\
\sin (\omega+\nu) \sin i
\end{array}\right),
$$

with

$$
r=\frac{a\left(1-e^{2}\right)}{1+e \cos v},
$$

where $\Omega$ is the longitude of the ascending node, $\omega$ is the argument of perigee, $v$ is the true anomaly, $i$ the inclination, $a$ is the semi-major axis and $e$ is the eccentricity. Using the geocentric coordinate system, the incident radiation vector is:

$$
\bar{\rho}_{s a t}=\rho_{x} e_{x}+\rho_{y} e_{y}+\rho_{z} e_{z}
$$

with

$$
\begin{aligned}
& \rho_{x}=r(\cos \Omega \cos (\omega+\mathrm{v})-\sin \Omega \sin (\omega+\mathrm{v}) \cos i)-G_{1} \cos \varphi_{g} \cos \theta, \\
& \rho_{y}=r(\sin \Omega \cos (\omega+\mathrm{v})+\cos \Omega \sin (\omega+\mathrm{v}) \cos i)-G_{1} \cos \varphi_{g} \sin \theta, \\
& \rho_{z}=r \sin (\omega+v) \sin i-G_{2} \sin \varphi_{g} .
\end{aligned}
$$

\subsection{Acceleration Components}

The acceleration experienced by a satellite of mass $M$ and cross sectional area $A$ is:

$$
\bar{a}=\frac{\bar{f}_{\rho}}{M}
$$

The radial components of the disturbing accelerations, $\tilde{S}$ is given by:

$$
\begin{aligned}
\tilde{S} & =\bar{a} \cdot \hat{r} \\
& =\frac{S}{c \rho_{s a t}} \frac{A}{M}\left[\left(\frac{3}{2} \beta \rho^{\prime}+\frac{1}{2}\left(B_{f}(1-\beta) \rho^{\prime}+\right.\right.\right. \\
& \left.\left.+\alpha^{\prime} \frac{\varepsilon_{f} B_{f}-\varepsilon_{b} B_{b}}{\varepsilon_{f}+\varepsilon_{b}}\right)^{2}+\frac{1}{2}\left(1-\beta \rho^{\prime}\right)^{2}\right)+\frac{3}{2}\left(1+\beta \rho^{\prime}\right)\left(B_{f}(1-\beta) \rho^{\prime}+\right. \\
& \left.+\alpha^{\prime} \frac{\varepsilon_{f} B_{f}-\varepsilon_{b} B_{b}}{\varepsilon_{f}+\varepsilon_{b}}\right) \cos \eta+\left(2 \beta \rho^{\prime}+\frac{1}{2}\left(B_{f}(1-\beta) \rho^{\prime}+\alpha^{\prime} \frac{\varepsilon_{f} B_{f}-\varepsilon_{b} B_{b}}{\varepsilon_{f}+\varepsilon_{b}}\right)^{2}+\right. \\
& \left.+\frac{1}{2}\left(1-\beta \rho^{\prime}\right)^{2}\right) \cos 2 \eta+\frac{1}{2}\left(1+\beta \rho^{\prime}\right)\left(B_{f}(1-\beta) \rho^{\prime}+\alpha^{\prime} \frac{\varepsilon_{f} B_{f}-\varepsilon_{b} B_{b}}{\varepsilon_{f}+\varepsilon_{b}}\right) \times \\
& \left.\times \cos 3 \eta+\frac{1}{2} \beta \rho^{\prime} \cos 4 \eta\right]^{1 / 2}\left[r-G_{1} \cos \varphi^{\prime}\left(\cos \left(\theta^{\prime}-\Omega\right) \cos (\omega+v)+\sin \left(\theta^{\prime}-\right.\right.\right. \\
& \left.-\Omega) \sin (\omega+v) \cos i)+G_{2} \sin \varphi^{\prime} \sin i \sin (\omega+v)\right] \cos \vartheta .
\end{aligned}
$$

The tangential component of disturbing acceleration is given by:

$$
\tilde{T}=\bar{a} \cdot(\hat{H} \times \hat{r})
$$

where $\hat{H}$ is a unit vector in the direction of the angular momentum vector. It can be expressed as [7]:

$$
\begin{aligned}
\hat{H} & =\sin i \sin \Omega e_{x}-\sin i \cos \Omega e_{y}+\cos i e_{z}, \\
\tilde{T} & =\frac{S}{c \rho_{s a t}} \frac{A}{M}\left[\left(\frac{3}{2} \beta \rho^{\prime}+\frac{1}{2}\left(B_{f}(1-\beta) \rho^{\prime}+\alpha^{\prime} \frac{\varepsilon_{f} B_{f}-\varepsilon_{b} B_{b}}{\varepsilon_{f}+\varepsilon_{b}}\right)^{2}+\right.\right. \\
& \left.+\frac{1}{2}\left(1-\beta \rho^{\prime}\right)^{2}\right)+\frac{3}{2}\left(1+\beta \rho^{\prime}\right)\left(B_{f}(1-\beta) \rho^{\prime}+\alpha^{\prime} \frac{\varepsilon_{f} B_{f}-\varepsilon_{b} B_{b}}{\varepsilon_{f}+\varepsilon_{b}}\right) \cos \eta+ \\
& +\left(2 \beta \rho^{\prime}+\frac{1}{2}\left(B_{f}(1-\beta) \rho^{\prime}+\alpha^{\prime} \frac{\varepsilon_{f} B_{f}-\varepsilon_{b} B_{b}}{\varepsilon_{f}+\varepsilon_{b}}\right)^{2}+\frac{1}{2}\left(1-\beta \rho^{\prime}\right)^{2}\right) \cos 2 \eta+ \\
& \left.+\frac{1}{2}\left(1+\beta \rho^{\prime}\right)\left(B_{f}(1-\beta) \rho^{\prime}+\alpha^{\prime} \frac{\varepsilon_{f} B_{f}-\varepsilon_{b} B_{b}}{\varepsilon_{f}+\varepsilon_{b}}\right) \cos 3 \eta+\frac{1}{2} \beta \rho^{\prime} \cos 4 \eta\right]^{1 / 2} \times \\
& \times\left[G_{1} \cos \varphi^{\prime}\left(\cos \left(\theta^{\prime}-\Omega\right) \sin (\omega+v)-\sin \left(\theta^{\prime}-\Omega\right) \cos (\omega+v) \cos i\right)-\right. \\
& \left.-G_{2} \sin \varphi^{\prime} \sin i \sin (\omega+v)\right] \cos \vartheta,
\end{aligned}
$$

The perpendicular component of disturbing acceleration is given by:

$$
\begin{aligned}
\tilde{W} & =\bar{a} \cdot \hat{H} \\
\tilde{W} & =\frac{S}{c \rho_{s a t}} \frac{A}{M}\left[\left(\frac{3}{2} \beta \rho^{\prime}+\frac{1}{2}\left(B_{f}(1-\beta) \rho^{\prime}+\alpha^{\prime} \frac{\varepsilon_{f} B_{f}-\varepsilon_{b} B_{b}}{\varepsilon_{f}+\varepsilon_{b}}\right)^{2}+\right.\right. \\
& \left.+\frac{1}{2}\left(1-\beta \rho^{\prime}\right)^{2}\right)+\frac{3}{2}\left(1+\beta \rho^{\prime}\right)\left(B_{f}(1-\beta) \rho^{\prime}+\alpha^{\prime} \frac{\varepsilon_{f} B_{f}-\varepsilon_{b} B_{b}}{\varepsilon_{f}+\varepsilon_{b}}\right) \cos \eta+ \\
& +\left(2 \rho_{s}^{\prime}+\frac{1}{2}\left(B_{f} \rho_{d}^{\prime}+\alpha^{\prime} \frac{\varepsilon_{f} B_{f}-\varepsilon_{b} B_{b}}{\varepsilon_{f}+\varepsilon_{b}}\right)^{2}+\frac{1}{2}\left(1-\rho_{s}^{\prime}\right)^{2}\right) \cos 2 \eta+\frac{1}{2}(1+ \\
& \left.\left.+\beta \rho^{\prime}\right)\left(B_{f}(1-\beta) \rho^{\prime}+\alpha^{\prime} \frac{\varepsilon_{f} B_{f}-\varepsilon_{b} B_{b}}{\varepsilon_{f}+\varepsilon_{b}}\right) \cos 3 \eta+\frac{1}{2} \beta \rho^{\prime} \cos 4 \eta\right]^{1 / 2} \times \\
& \times\left[G_{1} \cos \varphi^{\prime} \sin i \sin \left(\theta^{\prime}-\Omega\right)-G_{2} \sin \varphi^{\prime} \cos i\right] \cos \vartheta .
\end{aligned}
$$

It is worth noting that the spacecraft is decomposed into a finite number of small elementary surfaces. Consequently, 
the total force acting on the cubesat is considered to be the sum of all forces acting on each elementary surface [3], [10] and [14]. At a given time, the total radiant acceleration affecting the spacecraft is

$$
\bar{a}(t)=\sum_{i=1}^{k} \bar{a}_{i}(t)
$$

where $\mathrm{k}$ is the number of satellite surfaces and $\bar{a}_{i}(t)$.

\subsection{Albedo force on Cubesats}

The Cubesat is a cube shaped picosatellite constrained to CalPoly's specifications. The structure of these cubesats may be consisted of one cube " $1 U$ ", two cubes " $2 U$ " or three cubes "3U" as specified in table 1 [5] and [12].

The cubesat's thermo-optical properties are also constrained to CalPoly's specifications as tabulated in table2 [5] and [12]:

Based on Eqs. 15, 17 and 18 and make use of the thermooptical properties and area to mass ratio of cubesats, the acceleration components experienced by a cubesat are given by the following equations:

$$
\begin{aligned}
\tilde{S} & =\frac{C 1 S}{\rho_{\text {sat }}}\left[r-G_{1} \cos \varphi^{\prime}\left(\cos \left(\theta^{\prime}-\Omega\right) \cos (\omega+v)+\sin \left(\theta^{\prime}-\Omega\right) \sin (\omega+v) \cos i\right)+\right. \\
& \left.+G_{2} \sin \varphi^{\prime} \sin i \sin (\omega+v)\right] \cos \vartheta . \\
\tilde{T} & =\frac{C 1 S}{\rho_{\text {sat }}}\left[G_{1} \cos \varphi^{\prime}\left(\cos \left(\theta^{\prime}-\Omega\right) \sin (\omega+v)-\sin \left(\theta^{\prime}-\Omega\right) \cos (\omega+v) \cos i\right)-\right. \\
& \left.-G_{2} \sin \varphi^{\prime} \sin i \sin (\omega+v)\right] \cos \vartheta, \\
\tilde{W} & =\frac{C 1 S}{\rho_{\text {sat }}}\left[G_{1} \cos \varphi^{\prime} \sin i \sin \left(\theta^{\prime}-\Omega\right)-G_{2} \sin \varphi^{\prime} \cos i\right] \cos \vartheta .
\end{aligned}
$$

On account of the different area to mass ration of each cubesat, the constant $C 1=2.9 \times 10^{-9}$ is used for $1 \mathrm{U}$ cubesat, where $C 1=2.4 \times 10^{-9}$ is used for $2 \mathrm{U}$ cubesat and $C 1=1.8 \times 10^{-9}$ is used for $3 \mathrm{U}$ cubesat in case the radiation falls normal to the satellite surface (i.e. $\eta=0$ ).

\section{Perturbations of the orbital elements}

For orbits of $e \neq 0$ and $i \neq 0$, the effect of radiation pressure on the satellite's orbital elements; the semi-major axis $a$, the eccentricity $e$, the mean anomaly $M$, the inclination $i$, the longitude of the node $\Omega$ and the argument of the perigee $\omega$ can be evaluated using the general perturbation equations

\begin{tabular}{|c|c|c|c|c|}
\hline Component & Material & Thermal finishing & $\begin{array}{c}\text { Absorption } \\
\text { coefficient } \grave{\alpha}\end{array}$ & $\begin{array}{c}\text { Emissivity } \\
\varepsilon\end{array}$ \\
\hline Aluminium frame & $\begin{array}{l}\text { Aluminium } 5052 \\
\text { alloy }\end{array}$ & Alodine & 0.08 & 0.15 \\
\hline $\begin{array}{l}\text { Aluminium frame } \\
\text { rails }\end{array}$ & $\begin{array}{l}\text { Aluminium } 6061 \\
\text { or } 7075 \text { alloy }\end{array}$ & Hard anodized & 0.88 & 0.88 \\
\hline Inside Aluminium & Aluminium 7075 & Alodine & - & 0.1 \\
\hline panels & alloy & & & \\
\hline $\begin{array}{l}\text { Aluminium panels } \\
\text { outside }\end{array}$ & $\begin{array}{l}\text { Aluminium } 7075 \\
\text { alloy }\end{array}$ & Kepton foil & 0.87 & 0.81 \\
\hline Solar cells & $\begin{array}{c}\text { Triple junction } \\
\text { GaAs cells }\end{array}$ & Anti-reflecting coating & 0.91 & 0.81 \\
\hline PCBs & FR4 & - & - & .8 \\
\hline Battery Pack & Plastic & - & - & .8 \\
\hline
\end{tabular}
of Gauss [18].

The variation of $M$ contains both mean motion of the

Table 1. Area and mass of $1 \mathrm{U}$ and $3 \mathrm{U}$ cubesats

\begin{tabular}{|c|c|c|}
\hline The Cubesat configuration & Mass $(\mathrm{kg})$ & Dimensions \\
\hline $1 \mathrm{U}$ & 1 & $10 \mathrm{~cm} \times 10 \mathrm{~cm} \times 11.3 \mathrm{~cm}$ \\
\hline $2 \mathrm{U}$ & 2 & $10 \mathrm{~cm} \times 10 \mathrm{~cm} \times 22 \mathrm{~cm}$ \\
\hline $3 \mathrm{U}$ & 4 & $10 \mathrm{~cm} \times 10 \mathrm{~cm} \times 34 \mathrm{~cm}$ \\
\hline
\end{tabular}

Table 2. Main characteristics of the cubesat external structure 
osculating ellipse and the effect of the perturbations. So, to find an element that changes slowly with a time derivative going to zero, a new variable, $\varsigma$, will be defined as [18]:

$$
M(t)=\Psi+\varsigma, \quad \Psi=\int_{t_{o}}^{t} \widehat{n}(t) d t
$$

where $M(t)$ is the osculating mean anomaly at time $t$ and $\hat{n}$ is the mean motion. The perturbation equations are obtained as functions of the acceleration components $\tilde{S}, \tilde{T}$ and $\tilde{W}$ as the following [18]:

$$
\begin{aligned}
\frac{d a}{d t} & =\frac{2}{\hat{n} \sqrt{1-e^{2}}}[\tilde{T}+\tilde{S} e \sin v+\tilde{T} e \cos v], \\
\frac{d e}{d t} & =\frac{\sqrt{1-e^{2}}}{\hat{n} a}[\tilde{S} \sin v+\tilde{T}(\cos E+\cos v)], \\
\frac{d i}{d t} & =\frac{\tilde{W} \sqrt{1-e^{2}}}{\hat{n} a} \cos (\omega+v), \\
\frac{d \Omega}{d t} & =\frac{\tilde{W} \sqrt{1-e^{2}}}{\widehat{n} a \sin i} \sin (\omega+v), \\
\frac{d \omega}{d t} & =\frac{\sqrt{1-e^{2}}}{\widehat{n} a e}\left[-\tilde{S} \cos v+\tilde{T}\left(\sin v+\frac{1}{\sqrt{1-e^{2}}} \sin E\right]-\right. \\
& -\frac{\tilde{W} \sqrt{1-e^{2}}}{\hat{n} a} \sin (\omega+v) \cot i, \\
\frac{d \varsigma}{d t} & =\tilde{S}\left[\frac{\cos E}{\hat{n} a e\left(1-e^{2}\right)}-\frac{\sqrt{1-e^{2}} \sin v \sin E}{\hat{n} a}+2 \frac{(1-e \cos E)}{\hat{n} a}\right]+ \\
& +\tilde{T}\left[\frac{\sqrt{1-e^{2}} \sin E}{\hat{n} a e}\left(\frac{e \cos E}{1-e \cos E}\right)-\frac{\sqrt{1-e^{2}} \sin E}{\hat{n} a}(\cos v+\cos E)\right] \\
\frac{d^{2} \Psi}{d t^{2}} & =-\frac{3}{a \sqrt{1-e^{2}}}[\tilde{T}+\tilde{S} e \sin v+\tilde{T} e \cos v],
\end{aligned}
$$

where $\mathrm{E}$ is the eccentric anomaly.

\section{Numerical Application}

Having implemented the Earth's reflectivity data measured by NASA's Earth Probe satellite, which is part of the TOMS project (Total Ozone Mapping Spectrometer) [15], the Earth's reflectivity as a function of latitudes is illustrated in fig. 2.

As illustrated in Fig. 2, the albedo has a maximum value of $\sim 88 \%$ over the latitude of 88.75 South (close to the South Pole). However, it has a minimum value of $\sim 13.8 \%$ over the latitude of 1.25 South (close to the equator).

In the present work, the albedo force is calculated for

\begin{tabular}{|c|c|c|c|c|c|c|c|}
\hline Satellite & & $\begin{array}{l}\text { NORD } \\
\text { ID }\end{array}$ & $\begin{array}{c}\text { Perigee } \\
\text { height } \\
{[\mathrm{km}]}\end{array}$ & $\mathbf{i}$ & $\mathbf{E}$ & $\begin{array}{c}\text { Maximum Albedo } \\
\text { Force[N] }\end{array}$ & $\begin{array}{c}\text { Minimum Albedo } \\
\text { Force[N] }\end{array}$ \\
\hline XaTcobeo & (1U) & 38082 & 314.4 & $69.48^{\circ}$ & .068 & $1.6 \times 10^{-7}$ & $1.6 \times 10^{-9}$ \\
\hline SEED & (1U) & 32791 & 613.8 & $97.78^{\circ}$ & 0.01 & $2 \times 10^{-7}$ & $1.5 \times 10^{-9}$ \\
\hline CAPE 1 & (1U) & 31130 & 650.3 & $97.89^{\circ}$ & .010 & $1.2 \times 10^{-7}$ & $1.7 \times 10^{-9}$ \\
\hline CSTB 1 & (1U) & 31122 & 651.9 & $97.86^{\circ}$ & 0.084 & $5.5 \times 10^{-7}$ & $7.4 \times 10^{-9}$ \\
\hline SAUDICOMSAT 6 & (1U) & 31121 & 655.1 & $97.85^{\circ}$ & 0.079 & $5.2 \times 10^{-7}$ & $6.8 \times 10^{-9}$ \\
\hline SAUDICOMSAT 4 & $(1 \mathrm{U})$ & 31127 & 656.1 & $97.84^{\circ}$ & 0.069 & $3.7 \times 10^{-7}$ & $4.6 \times 10^{-9}$ \\
\hline SAUDICOMSAT 7 & (1U) & 31119 & 657.4 & $97.83^{\circ}$ & 0.061 & $2.6 \times 10^{-7}$ & $3 \times 10^{-9}$ \\
\hline SAUDICOMSAT 5 & (1U) & 31124 & 658.2 & $97.83^{\circ}$ & 0.052 & $4.8 \times 10^{-7}$ & $5.4 \times 10^{-9}$ \\
\hline SAUDICOMSAT 3 & (1U) & 31125 & 659.1 & $97.83^{\circ}$ & 0.043 & $2.7 \times 10^{-7}$ & $2.9 \times 10^{-9}$ \\
\hline BEESAT & $(1 \mathrm{U})$ & 35933 & 714.8 & $98.35^{\circ}$ & .075 & $4.4 \times 10^{-7}$ & $6.9 \times 10^{-10}$ \\
\hline Cute-1.7+APD & $(2 \mathrm{U})$ & 32785 & 616.7 & $97.78^{\circ}$ & .013 & $1.6 \times 10^{-7}$ & $1.2 \times 10^{-9}$ \\
\hline CANX-2 & $(3 \mathrm{U})$ & 32790 & 616.2 & $97.78^{\circ}$ & 0.014 & $1.3 \times 10^{-7}$ & $3.4 \times 10^{-9}$ \\
\hline MAST & (3U) & 31126 & 650.4 & $97.87^{\circ}$ & .094 & $2.8 \times 10^{-7}$ & $3.9 \times 10^{-9}$ \\
\hline QUAKESAT & $(3 \mathrm{U})$ & 27846 & 823.3 & $98.71^{\circ}$ & .083 & $7.9 \times 10^{-9}$ & $1.08 \times 10^{-10}$ \\
\hline
\end{tabular}
some LEO cubesats of different configuration and perigee heights. The results are tabulated in the following table:

As illustrated in table 1, the maximum albedo force is in the order of $\sim 10^{-7} \mathrm{~N}$ and the minimum value is in the order of $\sim 10^{-10} \mathrm{~N}$ which is experienced by a cubesat of $\sim 823$ perigee

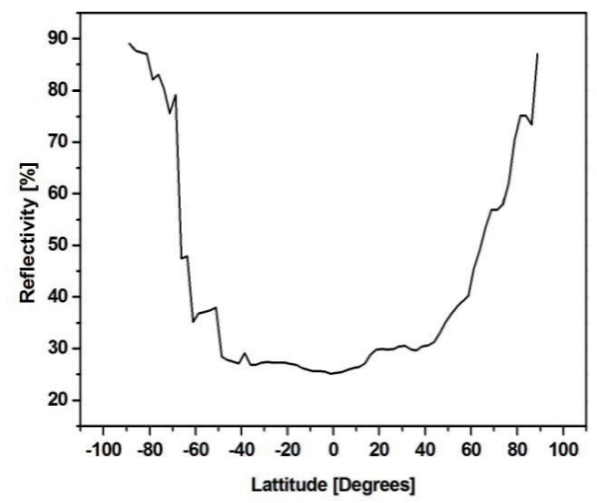

Fig. 2. Latitude dependency of Earth's reflectivity during the local summer

Table 3. Albedo force affecting on LEO cubesats of different perigee heights and area to mass ratio for one satellite revolution 
height.

The dependency of the albedo force on the orbital eccentricity is studied. Some cubesats are chosen approximately for the same inclination and different eccentricities and arranged in ascending order according

Table 4. Dependency of albedo force on the orbit eccentricity

\begin{tabular}{|c|c|c|c|c|}
\hline \multicolumn{2}{|l|}{ Satellite } & i & e & $\begin{array}{c}\text { Maximum Albedo } \\
\text { Force }[\mathbf{N}]\end{array}$ \\
\hline CSTB 1 & (1U) & $97.86^{\circ}$ & 0.084 & $1.2 \times 10^{-5}$ \\
\hline SAUDICOMSAT 6 & $(1 U)$ & $97.85^{\circ}$ & 0.079 & $1 \times 10^{-5}$ \\
\hline SAUDICOMSAT 4 & (1U) & $97.84^{\circ}$ & 0.069 & $3.6 \times 10^{-6}$ \\
\hline SAUDICOMSAT 7 & $(1 U)$ & $97.83^{\circ}$ & 0.061 & $1.2 \times 10^{-6}$ \\
\hline SAUDICOMSAT 5 & $(1 U)$ & $97.83^{\circ}$ & 0.052 & $4.8 \times 10^{-7}$ \\
\hline SAUDICOMSAT 3 & $(1 U)$ & $97.83^{\circ}$ & 0.043 & $2.7 \times 10^{-7}$ \\
\hline CAPE 1 & $(1 \mathrm{U})$ & $97.89^{\circ}$ & 0.010 & $9.1 \times 10^{-9}$ \\
\hline
\end{tabular}

to their eccentricities. The results showed that albedo force depends directly on the orbital eccentricity as seen in table 2 .

The mean orbital perturbations of the orbital elements are studied and illustrated in table 3 .

Based on the tabulated results in table 3 , the maximum perturbations of the semi-major axis, eccentricity and argument of perigee are in the order of $\sim 10^{-4}, 10^{-10}$ and $10^{-9}$, respectively. However, the minimum values are in the order of $\sim 10^{-6}, 10^{-12}$ and $10^{-11}$, respectively.

The perturbations of the semi-major axis, eccentricity and argument of perigee of the satellite XaTcobeo are illustrated in details over one revolution in the following figs 3- 5:

As illustrated previous figures, the maximum albedo perturbations for the semi-major axis, eccentricity and argument of perigee were experienced by the satellite when

Table 5. Mean orbital perturbations of some LEO cubesats due to albedo effects over one satellite revolution

\begin{tabular}{|l|c|c|c|c|c|}
\hline Satellite & $\dot{\bar{a}}$ & $\dot{\bar{e}}$ & $\dot{\bar{l}}$ & $\dot{\bar{\omega}}$ & $\dot{\bar{\Omega}}$ \\
\hline MAST & $1.9 \times 10^{-4}$ & $-1.5 \times 10^{-10}$ & $8.8 \times 10^{-18}$ & $-1.9 \times 10^{-9}$ & $-8.9 \times 10^{-19}$ \\
XaTcobeo & $-1.04 \times 10^{-4}$ & $-1.06 \times 10^{-10}$ & $4.8 \times 10^{-18}$ & $-6.5 \times 10^{-10}$ & $7.7 \times 10^{-21}$ \\
BEESAT & $-3.1 \times 10^{-4}$ & $-2.9 \times 10^{-10}$ & $-1 \times 10^{-20}$ & $-2 \times 10^{-10}$ & $1.6 \times 10^{-20}$ \\
CAPE 1 & $-1.07 \times 10^{-5}$ & $-7.4 \times 10^{-11}$ & $-1.4 \times 10^{-18}$ & $-2.7 \times 10^{-9}$ & $7.1 \times 10^{-19}$ \\
QUAKESAT & $-1.9 \times 10^{-6}$ & $-1.4 \times 10^{-12}$ & $1.1 \times 10^{-19}$ & $3.2 \times 10^{-11}$ & $-9.1 \times 10^{-21}$ \\
\hline
\end{tabular}

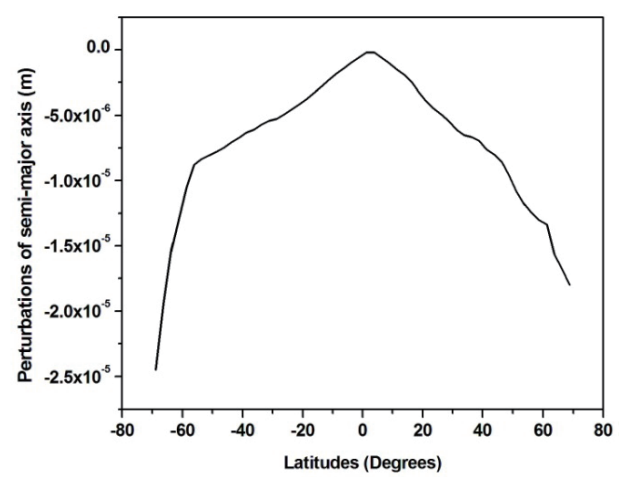

Fig. 3. Perturbation of the semi-major axis of XaTcobeo due to albedo effects over one satellite revolution

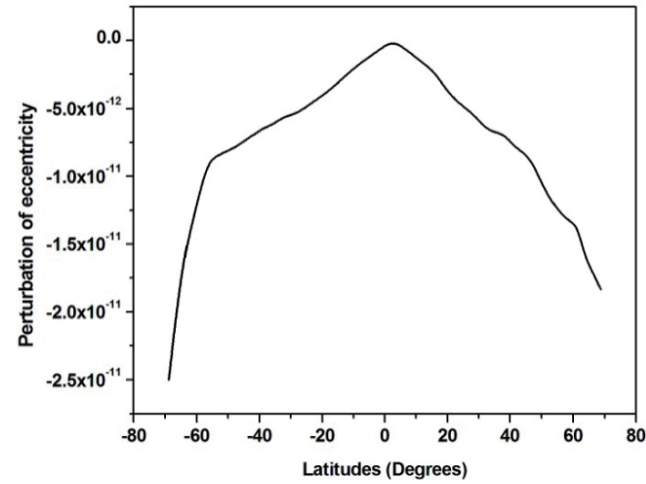

Fig. 4. Perturbation of the orbit eccentricity of XaTcobeo due to albedo effects over one satellite revolution

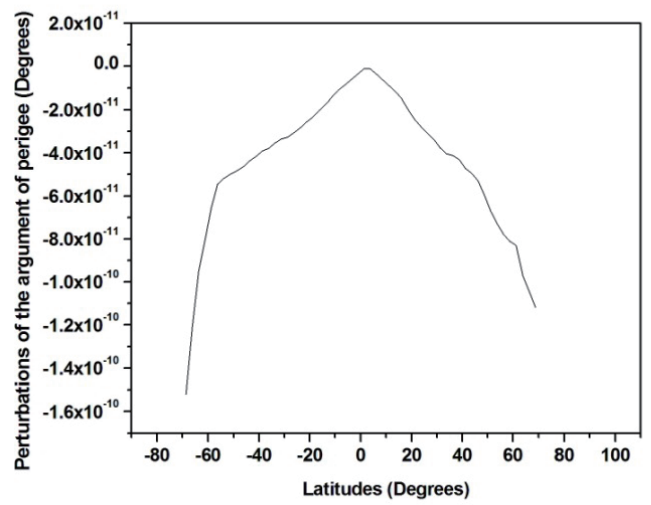

Fig. 5. Perturbation of the argument of perigee of XaTcobeo due to albedo effects over one satellite revolution 
it passes close to the Earths poles where the Earth's reflected radiation has its maximum percentage. However, the minimum perturbations occurred when the satellite passes close to the Earths equator.

\section{Conclusion}

A simple analytical model of the terrestrial albedo force was constructed w.r.t. the geocentric equatorial coordinate system in which the Earth was considered as an oblate spheroid.

The current numerical test has validated that the albedo force has significant contribution on the satellite dynamics and also a great dependency on the geodetic latitudes which depends on the satellite field of view. The maximum albedo force is in the order of $\sim 10^{-7} \mathrm{~N}$. Nevertheless, the minimum value is in the order of $\sim 10^{-10} \mathrm{~N}$.

The results show that the maximum perturbations of the semi-major axis, eccentricity and argument of perigee are in the order of $10^{-4}, 10^{-10}$ and $10^{-9}$, respectively. However, the minimum values are in the order of $10^{-6}, 10^{-12}$ and $10^{-11}$, respectively. Therefore, we can conclude that the effects of the albedo have a considerable disturbance on the orbit and it can affect the cubesats life time.

\section{Future work}

In our future work, the Sun's apparent path (the ecliptic) in the theoretical modelling and application will be considered. Moreover, the dependency of the albedo intensity on the Earth's longitude is to be investigated.

\section{References}

[1] Ali Ravanbakhsh, and Sebastian Franchini, "Preliminary Structural Sizing of a Modular Microsatellite Based on System Engineering Considerations", Third International Conference on Multidisciplinary Design Optimization and Applications, Paris, France, 2010.

[2] Anselmo L., Farinella P., Milani A., and Nobili, A. M.,, "Effects of the Earth Reflected Sunlight on the Orbit of the LAGEOs Satellite", Astronomy and Astrophysics, Vol 117, No. 1, 1983, 1-3.

[3] Antreasian, P. G. and Rosborough, G. W. , "Prediction of radiant energy forces on the TOPEX/POSEIDON spacecraft",
Journal of Spacecraft and Rockets, vol 2. No. 1, 1992, 81-90.

[4] Bhanderi D.D.V., "Spacecraft attitude determination with Earth albedo corrected Sun sensor measurements", Ph.D.Thesis, Aalborg University, Denmark, 2005.

[5] Bhanderi D.D.V. and Bak T., "Modeling Earth Albedo for Satellites in Earth Orbit", AIAA Guidance", Navigation, and Control Proceedings, San Francisco, California, 2005

[6] Cal Poly SLO, CubeSat Design Specification, URL : http://www.cubesat.org/images/developers/cds_rev12.pdf.

[7] Escoba P.R., Methods of Orbit Determination, John Wiley and Sons, Inc., New York, London, Sydney, 1965.

[8] Harris M., and Lyle R., "Spacecraft Radiation Torque," NASA Space Vehicle Design Criteria (Guidance and Control) NASA SP-8027, October, 1969.

[9] Jinsong Ping, Arata Sengoku, Nobuaki Nagaoka, Takahiro Iwata, Koji Matsumoto, and Nobuyuki Kawano", How solar radiation pressure acts on RSAT and VSAT with a small evolving tip-off in SELENE", Earth Planets Space, Vol 53, 2001, 919-925.

[10] Karla Patricia Vega, Attitude Control System for Cubesat for Ions, Neutrals, Electrons and MAGnetic Field (CINEMA), MS. Thesis, UNIVERSITY OF CALIFORNIA, BERKELEY,2009.

[11] Lionel Jacques, "Thermal Design of the Oufti-1 Nanosatellite", MS. thesis, University of Liège, 2009.

[12] Mc Innes Colin R., Solar Sailing: Technology, Dynamics and Mission Applications, Springer-Praxis Series in Space Science and Technology, Chichester, 1999.

[13] Milani, A., Nobili, A.M. and Farinella, P., Nongravitational perturbations and satellite geodesy", IOP publishing Ltd, 1987.

[14] NASA Total Ozone Mapping Spectrometer (TOMS Project), URL : http://toms.gsfc.nasa.gov/reflect/reflect v8.html

[15] Pontus Appel, "Attitude estimation from magnetometer and earth-albedo-corrected coarse Sun sensor measurements", Journal of Acta Astronautica, Vol. 56, Issue: 1-2, 2005.

[16] Rocco E. M., "Evaluation of the Terrestrial Albedo Applied to Some Scientific Missions", Space Science Review, Vol. 151, No. 1-3, 2009, pp. 135-147. DOI : 10.1007/s11214009-9622-6.

[17] Roy,A.E., Orbital motion, Adam Hilger Ltd, Bristol, 1978

[18] Venkataraman, N. S. and Carrara V, "The modeling of forces and torques on near Earth satellite application to a proposed Brazilian satellite", 7th Congr. Brasil. de Eng. Mecan., Uberlandia, Brazil, 1983. 